\title{
The Health System Costs of Potentially Inappropriate Prescribing: A Population-Based, Retrospective Cohort Study Using Linked Health Administrative Databases in Ontario, Canada
}

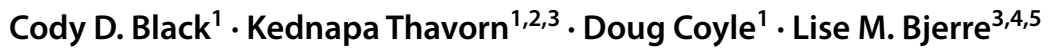

Published online: 19 June 2019

(c) The Author(s) 2019

\begin{abstract}
Objective The aim of this study was to determine the health system costs from hospitalizations, emergency department (ED) visits, and medications due to potentially inappropriate prescribing (PIP) in Ontario, Canada, at the population-level. Methods A retrospective cohort of individuals $\geq 66$ years of age and prescribed at least one medication from April 2002 to March 2015 was identified using linked population-level health administrative databases from Ontario, Canada. Patients were identified as having PIP or no PIP by applying a subset of the Screening Tool of Older Persons' Potentially Inappropriate Prescribing/Screening Tool to Alert Doctors to Right Treatment (STOPP/START) criteria. The number of days spent in hospital, new medications prescribed, and ED visits in the 90 days following PIP or patient index date were captured, as well as the total costs from each of these health services. Count regression models were used to generate incidence rate ratios (IRRs) for each outcome given the presence of PIP versus no PIP and combined with the prevalence of PIP to generate population attributable fractions (PAFs). The PAF was then multiplied by the cost for each health service to obtain the costs attributable to PIP in the whole cohort, and by age and sex. Results PIP was associated with an increased rate of hospitalization (IRR 2.77, 95\% confidence interval [CI] 2.72-2.82), ED visits (IRR 1.87, 95\% CI 1.82-1.92), and newly prescribed medications (IRR 1.13, 95\% CI 1.13-1.14), resulting in PAFs of 55.7, 37.9, and 5.0\% for each outcome, respectively. PIP was associated with $38.8 \%$ of the total spent on these healthcare services ( $\$ 1.22$ billion) in the 90 days after PIP. Costs attributable to PIP decreased with age despite increasing prevalence. Conclusions PIP in older adults is a significant source of health system costs from healthcare service use beyond medication costs, with a significant portion of hospitalizations and ED visit costs attributable to PIP. Future work should focus on identifying strategies and priorities for intervention.
\end{abstract}

Electronic supplementary material The online version of this article (https://doi.org/10.1007/s41669-019-0143-2) contains supplementary material, which is available to authorized users.

Lise M. Bjerre

lbjerre@uottawa.ca

1 School of Epidemiology and Public Health, University of Ottawa, Room 101, 600 Peter Morand Crescent, Ottawa, ON K1G 5Z3, Canada

2 Ottawa Hospital Research Institute, The Ottawa Hospital, 501 Smyth Box 511, Ottawa, ON K1H 8L6, Canada

3 ICES uOttawa, ICES, Administrative Services Building, 1st Floor, 1053 Carling Ave, Ottawa, ON K1Y 4E9, Canada

4 Department of Family Medicine, University of Ottawa, 600 Peter Morand Cresc. Suite 201, Ottawa, ON K1G 5Z3, Canada

5 Bruyère Research Institute, 43 Bruyère St., Ottawa, ON K1N 5C8, Canada

\section{Key Points for Decision Makers}

Potentially inappropriate prescribing (PIP) is not only a significant source of drug expenditures in older adults but also leads to increased costs from hospitalizations and emergency department (ED) visits.

PIP has the biggest impact on hospitalization costs, followed by ED visits and medications.

While PIP is more common as people age, it has a bigger impact on younger seniors. 


\section{Introduction}

Potentially inappropriate prescribing (PIP) describes the occurrence of prescribing practices that may increase a patient's risk for adverse health outcomes, given safer, more effective treatment options are available for a particular indication, or simply where the risks of therapy outweigh the benefits [1]. As a result, medication appropriateness in older persons is an important priority due to the potential for adverse drug events, increased morbidity, unplanned hospitalizations [2], emergency department (ED) visits [3] and mortality. In order to avoid negative outcomes such as these as a result of PIP, criteria have been developed, including the Screening Tool of Older Persons' Potentially Inappropriate Prescribing/Screening Tool to Alert Doctors to Right Treatment (STOPP/START) [4] and the Beers criteria [5], to help clinicians identify PIP in clinical settings and improve medication appropriateness.

Recently, subsets of both the STOPP/START and Beers criteria codable in large health administrative databases (HAD) have been applied to such data in Ontario housed at the ICES [6]. Through this initiative, the prevalence of PIP and its association with ED visits, hospitalization and mortality was identified. Instances of PIP were found to be frequent and were associated with all three of these outcomes [7]. Still, little is known about the health system costs associated with PIP at the population-level.

Previous studies have identified significant medication costs associated with PIP [8-10], while others have assessed the health system costs of specific PIP criterion [11]; however, to our knowledge, none have assessed the health system costs from ED visits and hospitalization due to PIP overall at the population-level. Contextualizing the health system cost burden of PIP, in conjunction with what we know about their association with health outcomes, may help inform policy discussions around the improvement of prescribing quality and medication appropriateness. They may also provide further evidence to various stakeholders to support the implementation of interventions at the health system level to support the broad improvement of medication appropriateness.

The aim of this study was to determine the health system costs from hospitalizations, ED visits, and newly prescribed medications due to PIP in Ontario, Canada, at the populationlevel. We also aimed to assess whether these costs differed between men and women, or between age groups.

\section{Methods}

The methods for this study, as well as their rationale, have been described extensively elsewhere in a study protocol [12]. As such, the methods are summarized below and follow the RECORD checklist [13]. This study was approved by the Institutional Review Board at Sunnybrook Health Sciences Centre, Toronto, ON, Canada.

\subsection{Study Design}

A population-based, retrospective cohort study design was used, spanning from 1 April 2002 to 31 March 2015. The patient accrual period was from 1 April 2003 to 31 December 2014, allowing for a 1-year lookback period for prior health services utilization and baseline covariates, as well as a 90-day follow-up period after the last possible PIP to allow time for identification of outcomes.

\subsection{Data Sources}

This study leveraged population-level health administrative datasets from Ontario housed at ICES [14, 15]. These datasets allowed for the linking of demographic, socioeconomic, hospital and outpatient health services, physician billing datasets, and prescription dispensation to all Ontarians aged 65 years or older with Ontario Health Insurance Plan (OHIP) coverage [16]. The following datasets were linked to gather desired exposure, outcome and covariate data necessary for analyses: Ontario Drug Benefits Claims Database (ODBD), Discharge Abstract Database (DAD), Same Day Surgery Database (SDS), National Ambulatory Care Reporting System (NACRS), OHIP database, and Registered Persons Database (RPDB) [17]. These datasets were linked using unique encoded identifiers and analyzed at ICES.

\subsection{Participants}

All Ontario residents aged 66 years of age or older with valid Ontario Drug Benefit coverage (approximately 97\% of Ontario seniors) and dispensed at least one prescription during the accrual period were eligible for the study. Patients aged 66 years or older were included to ensure 1-year lookback data, which were used to measure pre-medical history.

\subsection{Exposure}

The exposure of interest was the first occurrence of any PIP (yes/no) identified using health administrative data. Participants could experience multiple first PIPs from different criterion on the same day, although this only had bearing on the medication outcome models and certain sensitivity analyses, which are further described below. PIP were identified via the application of a subset of STOPP/START criteria, listed in Supplementary Appendix 1, applicable to health administrative databases [6]. This subset of criteria was coded into a format applicable to ICES-housed health administrative data using a combination of medication, diagnostic (i.e. 
International Classification of Diseases), healthcare services utilization and physician billing codes. The coding process is described in detail elsewhere [6].

\subsubsection{Assignment of Time to Potentially Inappropriate Prescribing (PIP) for Unexposed Patients}

To compare the outcomes of interest in participants with and without PIP, the index date in participants without PIP was treated as missing at random. We randomly assigned an index date to participants without PIP so that they would have comparable lookback and observation windows for covariate and outcome ascertainment, respectively.

Parametric survival analysis using a variety of distributions (i.e. normal, logistic, log-normal, log-logistic, exponential, gamma, Weibull) was conducted on participants who experienced PIP, to characterize their time-to-PIP, defined as the time from their accrual into the cohort until the time of the occurrence of their first PIP. Models were stratified by sex and median age during cohort participation, according to the following categories: $66-70$ years, $71-75$ years, 76-80 years, $81-85$ years, $86-90$ years, and over 90 years. The distribution, shape and scale parameters from the bestfitting models were then used with the random number function in SAS [18] in order to randomly assign a time-to-PIP to participants without PIP, based on their age and sex. This was then converted to an index date by adding the time-toPIP to the participant's accrual date. Participants assigned an index date that fell beyond the end of their follow-up period were excluded from analyses, while all participants, with or without PIP, who had an observation window smaller than 90 days were excluded from all analyses. Distribution of the time-to-index date between participants with and without PIP was assessed following the imputation process using a visual check of distributions, as well as a comparison of the mean time-to-index date between exposed and unexposed groups. The reason for this method of assignment of an index date to participants without PIP has been described in further detail in the study protocol [12].

\subsection{Outcome}

The primary outcome of interest was the combined medication, hospitalization and ED visit costs (reasons for the selection of these costs are available in the study protocol [12]) attributable to PIP paid by the Ontario Ministry of Health and Long-Term Care in the 90-day period following the first PIP occurrence for participants with PIP, or the index date for unexposed participants. These costs were assessed both in total for the whole cohort and per individual (an outcome not included in the study protocol). To estimate these costs, the ICES costing algorithm was used [19], which calculates the cost of each health service category by multiplying units of utilization for a health care service of interest over the investigator-defined period of time, by its unit cost. Hospitalization and ED costs were estimated by multiplying the weighted volume of services by the average provincial costs per weighted case. Medication costs were calculated based on the amount paid per prescription. All costs were inflated to 2017 Canadian dollars using the Statistic Canada's Consumer Price Index [20]. For participants not experiencing PIP, the 90-day follow-up period began on the date of random index date assignment, described in further detail above.

\subsection{Covariates}

All analyses were adjusted for potential confounders, either known or perceived to be associated with PIP, or with our outcomes of interest (i.e. hospitalization, ED visits and medication use) and available within our linked health administrative datasets, including age [21, 22], sex [22], neighbourhood income quintile [22, 23], rurality, Aggregated Diagnosis Group (ADG) score (i.e. comorbidity status) [22, 24, 25], number of unique drug identification numbers (i.e. pills prescribed concurrently) in the year prior to PIP [26-30], number of prescribers in the year prior to PIP [31], whether the patient had a MedsCheck (billable medication review performed by a pharmacist, usually in the community) in the year prior to PIP, number of days spent in hospital in the year prior to PIP, and ED visit in the 6 months prior to PIP [21, 22, 24, 32-36]. Definitions for these variables have been previously described elsewhere [37]. Covariate ascertainment was performed at the date of a participant's first PIP, and at the randomly assigned index date for participants who did not experience PIP.

\subsection{Statistical Analyses}

\subsubsection{Medication Use, Emergency Department Visits and Hospitalizations Attributable to PIP}

Once outcome and baseline covariate information were gathered for all participants, three models were created, one for each outcome: count of ED visits, days spent in hospital and total number of unique, newly prescribed medications. Prior to fitting each model, we tested for overdispersion to determine whether a Poisson or negative binomial distribution was most appropriate for our count regression models [38]. The incidence rate ratio (IRR) obtained via the parameter estimate for the PIP variable in the model for each outcome was used in conjunction with the population prevalence of a first PIP ever, to derive the population attributable fraction (PAF) via Levin's formula: $\left(\left(P_{\exp } \times(\mathrm{RR}-1)\right) /\right.$ $\left.\left(\left(P_{\exp }(\mathrm{RR}-1)\right)+1\right)\right) \times 100[39,40]$. This formula divides the product of the population prevalence multiplied by the 
IRR minus one, by the sum of one plus the product of the population prevalence multiplied by the IRR minus one. This figure is then multiplied by 100 to obtain a percentage. For the medication outcome model, only patients with PIP due to a STOPP criterion, and no overlapping, or multiple first, PIPs, were included to ensure only a potential error of commission was responsible as we were unable to determine what the exact first PIP was in such cases.

\subsubsection{Total Costs Attributable to PIP}

PAFs for ED visits, days spent in hospital and newly prescribed medications were then multiplied by the total costs for each of these health services, to obtain the cost of each health service use attributable to PIP over the 90-day observation period after PIP or randomly assigned index date. These costs were also combined to obtain the total health system costs attributable to PIP.

\subsubsection{Subgroup and Sensitivity Analyses}

The methods listed above were used to obtain the costs attributable to PIP by age, categorized as described above, and sex. Sensitivity analyses were also conducted, including the assignment of two additional random index dates to persons without PIP, to determine whether the random assignment method potentially biased study results, as well as the removal of people with their first PIP occurring on their date of entry into the cohort, to assess for the presence of incidence-prevalence bias due to the potential that these were prevalent and not incident PIPs. Additionally, the removal of people with multiple first PIPs and overlapping PIP during their observation period, two of the conditions applied to the inclusion of participants in the newly prescribed medications models, was applied to the hospitalization and ED visit outcome models to assess the impact of the first PIP alone on those outcomes. Finally, an analysis stratified by type of first PIP (i.e. STOPP [PIP] or START [PIO]) was also conducted to determine the impact of PIP type on hospitalization and ED visits.

\section{Results}

\subsection{Baseline Characteristics}

A total of 2,477,122 residents of Ontario prescribed at least one medication between 1 April 2003 and 31 December 2014 were identified. After assigning a random index date to persons without PIP, the cohort was further restricted to $2,256,153$ total study participants with at least 90 days of follow-up for outcome ascertainment (see Fig. A1 in Supplementary Appendix 2 for a description of how the final study cohort was obtained). Among included participants, the time-to-index date was well-balanced between participants with and without PIP after random index date assignment.

Among the identified cohort, the mean age was 72.1 years (standard deviation 7.4 ) and $44.8 \%$ were male $(1,010,761 / 2,256,163)$ (Table 1). When comparing baseline characteristics between the two groups, patients with PIP were, on average, older, had more comorbidity (identified via ADG), concurrent medications, days spent in hospital and prescribers, and were more likely to have a previous ED visit and to be in the lowest income quintile. No missing outcome data and very little missing covariate data were identified, with all of the data pertaining to either place of residence or income quintile. Data for these variables were missing for $<0.4 \%$ of participants.

\subsection{PIP Prevalence by Age and Sex}

After assessment of baseline characteristics, we further assessed PIP prevalence and prevalence of a first PIP due to a STOPP criterion only, in the whole cohort, as well as by age and sex. While $70.1 \%(1,581,897 / 2,256,163)$ of cohort members had at least one PIP, only 40.2\% $(907,993 / 2,256,163)$ of cohort members had a first PIP due to a STOPP criterion (Table 2). The overall prevalence of PIP remained similar between males and females, although females had a higher proportion of first PIPs due to a single STOPP criterion, indicating males had a higher prevalence of a first PIP due to a START criterion or multiple first PIPs. With regard to age, overall PIP prevalence tended to increase with age, from $57.0 \%(592,532 / 929,009)$ in the $66-70$ years age group, to $84.2 \%(215,660 / 256,025)$ in the $81-85$ years age group, and then remaining consistent thereafter, while the number of first PIPs due to a STOPP criterion tended to decrease with age.

\subsection{Costs Attributable to PIP in the Whole Cohort}

The total costs from hospitalizations, ED visits and newly prescribed medications from 1 April 2003 to 31 December 2014 in the 90 days after the index date were $\$ 2.00$ billion, $\$ 172$ million and $\$ 975$ million for each outcome, respectively; PIP was associated with increased rates for all three outcomes (Table 3). Participants with PIP had a 2.77 (95\% confidence interval [CI] 2.72-2.82) times higher rate of hospitalization than those without PIP, leading to a PAF of $55.3 \%$ (95\% CI 54.7-56.1). Increased rates of ED visits and newly prescribed medications were also observed in participants with PIP versus those without, with 1.87 (95\% CI 1.82-1.92) and 1.13 (95\% CI 1.13-1.14) times higher rates, respectively. When combined with the PIP prevalence, PAFs of $37.9 \%$ (95\% CI 36.5-39.2) for ED visits and 5.0\% (95\% CI 5.0-5.3) for newly prescribed medications were observed. 
Table 1 Characteristics of the study population

\begin{tabular}{|c|c|c|c|}
\hline Characteristic & $\mathrm{PIP}[N=1,581,897]$ & No PIP $[N=674,266]$ & $\begin{array}{l}\text { Primary analy- } \\
\text { sis cohort } \\
{[N=2,256,163]}\end{array}$ \\
\hline Mean age, years $(\mathrm{SD})$ & $74.0(7.6)$ & $69.8(5.8)$ & $72.1(7.4)$ \\
\hline Male sex $(\%)$ & 44.6 & 45.5 & 44.8 \\
\hline Number of ADGs (SD) & $9.2(3.7)$ & $6.3(3.4)$ & $8.3(3.7)$ \\
\hline Number of concurrent medications (SD) & $5.0(3.1)$ & $2.1(2.1)$ & $4.2(3.1)$ \\
\hline $\begin{array}{l}\text { Mean number of days spent in hospital in the year prior to index or } \\
\text { assigned index date (SD) }\end{array}$ & $3.6(11.6)$ & $0.6(4.8)$ & $2.7(10.1)$ \\
\hline$\geq 1$ ED visits in the 6 months prior to the index or assigned index date (\%) & 32.0 & 10.3 & 25.5 \\
\hline \multicolumn{4}{|l|}{ Income quintile (\%) } \\
\hline 1-lowest & 20.1 & 16.4 & 19.0 \\
\hline 2 & 20.9 & 19.6 & 20.5 \\
\hline 3 & 19.5 & 19.8 & 19.6 \\
\hline 4 & 19.4 & 20.9 & 19.9 \\
\hline 5-highest & 19.6 & 23.0 & 20.6 \\
\hline Missing & 0.4 & 0.4 & 0.4 \\
\hline Urban place of residence $^{\mathrm{a}}(\%)$ & 85.1 & 85.2 & 85.1 \\
\hline Number of prescribers in the year prior to the first PIP (SD) & $2.7(2.1)$ & $1.9(1.4)$ & $2.5(1.9)$ \\
\hline MedsCheck in the year prior $(\%)$ & 7.6 & 7.5 & 7.6 \\
\hline
\end{tabular}

$A D G$ Aggregate Diagnosis Group, $E D$ emergency department, $P I P$ potentially inappropriate prescription, $S D$ standard deviation ${ }^{\mathrm{a}} 0.11 \%$ of the cohort had missing information on place of residence

Table 2 Prevalence of PIP among the whole cohort and by subgroups

\begin{tabular}{lcll}
\hline Group & $\begin{array}{l}\text { No. of persons with a PIP } \\
\text { (STOPP criterion as the first PIP) }\end{array}$ & $\begin{array}{l}\text { No. of persons eligible } \\
\text { for the study cohort }\end{array}$ & $\begin{array}{l}\text { Prevalence (STOPP } \\
\text { criterion only) (\%) }\end{array}$ \\
\hline $\begin{array}{l}\text { Whole cohort } \\
\text { (STOPP criterion } \\
\text { only) }\end{array}$ & $1,581,897(907,993)$ & $2,256,163$ & $70.1(40.2)$ \\
Sex & & & \\
Female & & & \\
Male & $876,808(546,190)$ & $1,244,562$ & $70.5(43.9)$ \\
Median age during & $705,089(361,803)$ & $1,011,601$ & $69.7(35.8)$ \\
cohort participa- & & & \\
tion, years & & & \\
66-70 & $529,532(377,006)$ & 929,009 & $57.0(40.6)$ \\
$71-75$ & $393,177(218,908)$ & 529,320 & $74.3(41.4)$ \\
$76-80$ & $283,494(138,957)$ & 350,753 & $80.8(39.6)$ \\
$81-85$ & $215,660(100,369)$ & 256,025 & $84.2(39.2)$ \\
$86-90$ & $112,228(51,125)$ & 132,145 & $84.1(38.7)$ \\
$>90$ & $47,806(21,628)$ & 58,911 & $81.2(36.7)$ \\
\hline
\end{tabular}

PIP potentially inappropriate prescription, STOPP Screening Tool of Older People's Prescriptions
After multiplying the costs for hospitalization, ED visits and newly prescribed medications in the 90-day followup period by their respective PAFs, a total of $\$ 1.22$ billion of $\$ 3.15$ billion (38.8\%) from all three health services were attributable to PIP, with the highest proportion of costs stemming from hospitalizations, and the fewest stemming from new medications. The total cost from all three healthcare services per individual with PIP was $\$ 772.83$ (95\% CI 761.36-784.19).

\subsection{Costs Attributable to PIP by Age and Sex}

When assessing the costs attributable to PIP between males and females, few differences were observed, although PIP was still associated with higher rates of outcomes in both 
Table 3 Primary analysis: costs attributable to PIP

\begin{tabular}{|c|c|c|c|c|c|c|c|}
\hline $\begin{array}{l}\text { Health service/ } \\
\text { cost category }\end{array}$ & $\begin{array}{l}\text { No. of par- } \\
\text { ticipants with } \\
\text { PIP }\end{array}$ & $\begin{array}{l}\text { IRR with PIP vs. } \\
\text { no PIP (95\% CI) }\end{array}$ & $\begin{array}{l}\text { PIP } \\
\text { preva- } \\
\text { lence }(\%)\end{array}$ & $\begin{array}{l}\text { Population attrib- } \\
\text { utable fraction [\% } \\
(95 \% \mathrm{CI})]\end{array}$ & $\begin{array}{l}\text { Total costs }(2017 \\
\text { CAN\$) }\end{array}$ & $\begin{array}{l}\text { Costs attribut- } \\
\text { able to PIP } \\
(2017 \text { CAN\$) }\end{array}$ & $\begin{array}{l}\text { Cost per indi- } \\
\text { vidual }[2017 \text { CAN\$ } \\
(95 \% \mathrm{CI})]\end{array}$ \\
\hline Hospitalization & $1,581,897$ & $2.77(2.72-2.82)$ & 70.1 & $55.3(54.7-56.1)$ & $2,000,286,464$ & $1,107,608,294$ & $\begin{array}{l}699.97(691.01- \\
708.66)\end{array}$ \\
\hline ED visits & $1,581,897$ & $1.87(1.82-1.92)$ & 70.1 & $37.9(36.5-39.2)$ & $172,289,790$ & $65,268,856$ & $\begin{array}{l}41.24(39.74- \\
42.69)\end{array}$ \\
\hline \multirow[t]{2}{*}{ Medication $^{\mathrm{a}}$} & 907,993 & $1.13(1.13-1.14)$ & 40.2 & $5.0(5.0-5.3)$ & $975,197,362$ & $48,432,720$ & $\begin{array}{l}53.32(53.32- \\
57.21)\end{array}$ \\
\hline & & & & Total & $3,147,773,616$ & $1,221,309,870$ & $\begin{array}{l}772.83(761.36- \\
784.19)\end{array}$ \\
\hline
\end{tabular}

$C I$ confidence interval, $C A N \$$ Canadian dollars, $P I P$ potentially inappropriate prescription, IRR incidence rate ratio, $E D$ emergency department, STOPP Screening Tool of Older People's Prescriptions, START Screening Tool to Alert Doctors to Right Treatment

${ }^{\text {a } I n c l u d e s ~ o n l y ~ t h o s e ~ w i t h ~ a ~ S T O P P ~ c r i t e r i o n ~ a s ~ t h e i r ~ f i r s t ~ P I P . ~ A l l ~ p a r t i c i p a n t s ~ w i t h ~ a ~ S T A R T ~ c r i t e r i o n ~ a s ~ t h e i r ~ f i r s t ~ P I P, ~ m u l t i p l e ~ f i r s t ~ P I P, ~ o r ~ a n ~}$ overlapping second PIP within their 90-day outcome observation window were removed and the prevalence was recalculated

${ }^{\mathrm{b}}$ Costs attributable to PIP were determined by multiplying the unrounded population attributable fraction by the total costs

subgroups (Table 4). Males with PIP tended to have higher rates of each outcome than females with PIP when compared with participants without PIP of their respective sex; the observed difference in total costs attributable to PIP and cost per person with PIP is likely due to the higher PAF of PIP for hospitalization costs in males.

Assessment of the costs attributable to PIP by age category displays a trend for prevalence and PAF. While the prevalence of PIP tends to increase with age, the PAF tends to decrease with age due to a general trend of decreasing rates of outcomes due to PIP by age category. For example, while the youngest age category (66-70 years) had a 57\% PIP prevalence (40.6\% STOPP only) versus $81.2 \%$ (36.7\% STOPP only) for the oldest age category $(90+$ years), the PAFs for hospitalization, ED visits and newly prescribed medications were $55.2,33.7$ and $3.5 \%$, respectively, for the youngest age group, versus $34.2,28.5$ and $2.9 \%$, respectively, for the oldest age group. Additionally, due to the larger population size of the youngest age group, the absolute costs to the healthcare system from the outcomes of interest due to PIP are much larger (\$351 million) compared with the oldest age group (\$27 million).

\subsection{Sensitivity Analyses}

The random index date assignment had a limited impact on study results as the IRR estimates remained stable when the second and third random index date assignments were assessed, and they were within $10 \%$ of the primary analysis IRRs (Table A2 in Supplementary Appendix 3). Comparable results were also observed when assessing the removal of participants whose first PIP occurred on their first day of accrual into the study cohort to ensure no incidence-prevalence bias was present in our analyses. Changes in the IRRs for hospitalizations, from 2.77 (95\% CI 2.72-2.82) to 2.03 (95\% CI 1.98-2.08), and ED visits, from 1.87 (95\% CI $1.82-1.92)$ to 1.58 (95\% CI 1.57-1.60), were observed after the removal of participants with multiple first PIPs and overlapping PIPs within their outcome observation period. When comparing the IRRs, when restricting the cohort to participants with a STOPP criterion as their first PIP versus those with a START criterion as their first PIP, higher rates of hospitalizations and ED visits were observed in participants with a STOPP criterion.

\section{Discussion}

The results presented within this article describe the burden of PIP, further enhancing our understanding of this prevalent issue beyond the tabulation of medication costs, using a large cohort of older persons in Ontario identified using linked health administrative databases. We found that older persons with PIP experience higher rates of hospitalization, ED visits, and newly prescribed medications than their counterparts who have also been prescribed a medication but have not experienced PIP. When combined with the high prevalence of PIP in the population, PIP was associated with a significant portion of healthcare expenditures related to these outcomes.

Beyond the identification of PIP as a high-cost issue, this study has important findings with policy implications. Much of the discussion around medication appropriateness and PIP centres on the cost of the offending drugs themselves, but what we found is that the medication costs likely have a lower impact on healthcare expenditures than hospitalizations and ED visits. Not only would reducing PIP decrease drug expenditures but it could also prevent significant morbidity that leads to even larger downstream expenditures via lengthy hospital stays and unnecessary ED 
Table 4 Costs attributable to PIP, by sex and median age during cohort participation

\begin{tabular}{|c|c|c|c|c|c|c|c|}
\hline $\begin{array}{l}\text { Health service/cost } \\
\text { category }\end{array}$ & $\begin{array}{l}\text { No. of par- } \\
\text { ticipants with } \\
\text { PIP }\end{array}$ & $\begin{array}{l}\text { IRR with PIP vs. } \\
\text { no PIP }(95 \% \text { CI })\end{array}$ & $\begin{array}{l}\text { PIP } \\
\text { preva- } \\
\text { lence }(\%)\end{array}$ & $\begin{array}{l}\text { Population attrib- } \\
\text { utable fraction [\% } \\
(95 \% \mathrm{CI})]\end{array}$ & $\begin{array}{l}\text { Total costs }(2017 \\
\text { CAN\$) }\end{array}$ & $\begin{array}{l}\text { Costs } \\
\text { attributable } \\
\text { to PIP }\end{array}$ & $\begin{array}{l}\text { Cost per PIP per } \\
\text { individual [2017 } \\
\text { CAN\$ }(95 \% \mathrm{CI})]\end{array}$ \\
\hline \multicolumn{8}{|l|}{ Sex } \\
\hline \multicolumn{8}{|l|}{ Female } \\
\hline Hospitalization & 876,808 & $2.74(2.67-2.82)$ & 70.5 & $55.1(54.1-56.2)$ & $981,015,469$ & $540,446,255$ & $\begin{array}{l}616.20(604.81- \\
628.610\end{array}$ \\
\hline ED visits & 876,808 & $1.76(1.73-1.79)$ & 70.5 & $38.0(36.6-39.3)$ & $91,389,084$ & $34,743,543$ & $39.61(38.17-40.99)$ \\
\hline \multirow[t]{2}{*}{ Medication $^{\mathrm{a}}$} & 546,190 & $1.12(1.12-1.13)$ & 43.9 & $5.0(5.0-5.4)$ & $529,162,647$ & $26,481,255$ & $48.47(48.47-52.29)$ \\
\hline & & & & Total & $1,601,567,201$ & $601,671,053$ & $\begin{array}{l}686.01(673.18- \\
702.18)\end{array}$ \\
\hline \multicolumn{8}{|l|}{ Male } \\
\hline Hospitalization & 705,089 & $2.81(2.74-2.89)$ & 69.7 & $55.8(54.8-56.8)$ & $1,019,270,993$ & $568,579,220$ & $\begin{array}{l}806.16(792.07- \\
821.54)\end{array}$ \\
\hline ED visits & 705,089 & $1.84(1.81-1.87)$ & 69.7 & $36.9(36.1-37.7)$ & $80,900,705$ & $26,272,393$ & $42.36(41.39-43.30)$ \\
\hline \multirow[t]{2}{*}{ Medication $^{\mathrm{a}}$} & 361,803 & $1.15(1.14-1.15)$ & 35.8 & $5.1(4.8-5.1)$ & $446,034,717$ & $22,731,389$ & $62.81(58.82-62.81)$ \\
\hline & & & & Total & $1,546,206,415$ & $621,185,312$ & $\begin{array}{l}880.75(863.64- \\
897.06)\end{array}$ \\
\hline \multicolumn{8}{|l|}{ Age } \\
\hline \multicolumn{8}{|l|}{$66-70$ years } \\
\hline Hospitalization & 529,532 & $3.16(3.06-3.26)$ & 57.0 & $55.2(55.1-54.0)$ & $580,137,432$ & $320,126,033$ & $\begin{array}{l}604.37(591.50- \\
616.60)\end{array}$ \\
\hline ED visits & 529,532 & $1.89(1.86-1.92)$ & 57.0 & 33.7 (32.9-34.4) & $53,601,957$ & $18,040,386$ & $34.06(33.29-34.81)$ \\
\hline \multirow[t]{2}{*}{ Medication $^{\mathrm{a}}$} & 377,006 & $1.09(1.09-1.10)$ & 40.6 & $3.5(3.5-3.9)$ & $358,392,982$ & $12,634,032$ & $33.50(33.50-37.08)$ \\
\hline & & & & Total & $992,132,371$ & $350,800,451$ & $\begin{array}{l}662.28(648.64- \\
677.81)\end{array}$ \\
\hline \multicolumn{8}{|l|}{$71-75$ years } \\
\hline Hospitalization & 393,177 & $2.31(2.21-2.40)$ & 74.3 & $49.3(47.3-51.0)$ & $445,287,220$ & $219,634,531$ & $\begin{array}{l}558.45(536.00- \\
577.26)\end{array}$ \\
\hline ED visits & 393,177 & $1.69(1.63-1.74)$ & 74.3 & $33.9(31.9-35.4)$ & $35,881,151$ & $12,160,742$ & $30.92(29.09-32.36)$ \\
\hline \multirow[t]{2}{*}{ Medication $^{\mathrm{a}}$} & 218,908 & $1.22(1.21-1.23)$ & 41.4 & $8.3(8.0-8.7)$ & $216,478,481$ & $18,070,957$ & $82.52(79.08-85.95)$ \\
\hline & & & & Total & $697,646,851$ & $249,866,231$ & $\begin{array}{l}635.32(609.12- \\
657.48)\end{array}$ \\
\hline \multicolumn{8}{|l|}{$76-80$ years } \\
\hline Hospitalization & 283,494 & $2.14(2.04-2.25)$ & 80.8 & $47.9(45.7-50.2)$ & $411,617,375$ & $190,998,074$ & $\begin{array}{l}695.96(662.79- \\
729.37)\end{array}$ \\
\hline ED visits & 283,494 & $1.59(1.53-1.65)$ & 80.8 & $32.2(30.0-34.4)$ & $32,393,464$ & $10,457,373$ & $36.87(34.25-39.34)$ \\
\hline \multirow[t]{2}{*}{ Medication $^{\mathrm{a}}$} & 138,957 & $1.17(1.16-1.18)$ & 39.6 & $6.3(6.0-6.7)$ & $169,187,105$ & $10,671,285$ & $76.77(72.53-80.99)$ \\
\hline & & & & Total & $613,197,944$ & $211,445,822$ & $\begin{array}{l}770.47(732.59- \\
808.40)\end{array}$ \\
\hline \multicolumn{8}{|l|}{$81-85$ years } \\
\hline Hospitalization & 215,660 & $1.83(1.73-1.94)$ & 84.2 & $41.1(38.1-44.2)$ & $323,496,810$ & $133,076,875$ & $\begin{array}{l}616.89(570.86- \\
662.53)\end{array}$ \\
\hline ED visits & 215,660 & $1.61(1.56-1.67)$ & 84.2 & $33.9(32.0-36.1)$ & $27,778,415$ & $9,426,111$ & $43.70(41.26-46.44)$ \\
\hline \multirow[t]{2}{*}{ Medication $^{\mathrm{a}}$} & 100,369 & $1.13(1.12-1.14)$ & 39.2 & $4.8(4.5-5.2)$ & $132,504,575$ & $6,425,014$ & $64.00(59.29-68.66)$ \\
\hline & & & & Total & $483,779,798$ & $148,928,001$ & $\begin{array}{l}690.37(639.72- \\
740.92)\end{array}$ \\
\hline \multicolumn{8}{|l|}{$86-90$ years } \\
\hline Hospitalization & 112,228 & $1.79(1.66-1.93)$ & 84.1 & $39.9(35.7-43.9)$ & $168,275,404$ & $67,172,054$ & $\begin{array}{l}598.35(535.04- \\
657.86)\end{array}$ \\
\hline ED visits & 112,228 & $1.51(1.46-1.59)$ & 84.1 & $30.0(27.9-33.2)$ & $15,797,747$ & $4,741,944$ & $42.24(39.25-46.67)$ \\
\hline \multirow[t]{2}{*}{ Medication $^{\mathrm{a}}$} & 51,125 & $1.11(1.10-1.12)$ & 38.7 & $4.1(3.7-4.4)$ & $69,661,327$ & $2,844,397$ & $55.62(50.75-60.45)$ \\
\hline & & & & Total & $253,734,477$ & $74,758,395$ & $\begin{array}{l}665.94(597.41- \\
732.07)\end{array}$ \\
\hline
\end{tabular}


Table 4 (continued)

\begin{tabular}{|c|c|c|c|c|c|c|c|}
\hline $\begin{array}{l}\text { Health service/cost } \\
\text { category }\end{array}$ & $\begin{array}{l}\text { No. of par- } \\
\text { ticipants with } \\
\text { PIP }\end{array}$ & $\begin{array}{l}\text { IRR with PIP vs. } \\
\text { no PIP (95\% CI) }\end{array}$ & $\begin{array}{l}\text { PIP } \\
\text { preva- } \\
\text { lence }(\%)\end{array}$ & $\begin{array}{l}\text { Population attrib- } \\
\text { utable fraction [\% } \\
(95 \% \mathrm{CI})]\end{array}$ & $\begin{array}{l}\text { Total costs }(2017 \\
\text { CAN\$) }\end{array}$ & $\begin{array}{l}\text { Costs } \\
\text { attributable } \\
\text { to } \mathrm{PIP}^{\mathrm{b}}\end{array}$ & $\begin{array}{l}\text { Cost per PIP per } \\
\text { individual [2017 } \\
\text { CAN\$ }(95 \% \text { CI)] }\end{array}$ \\
\hline \multicolumn{8}{|l|}{$>90$ years } \\
\hline Hospitalization & 47,806 & $1.64(1.48-1.82)$ & 81.2 & $34.2(28.0-40.0)$ & $71,472,223$ & $24,441,122$ & $\begin{array}{l}511.11(419.17- \\
597.40)\end{array}$ \\
\hline ED visits & 47,806 & $1.49(1.41-1.58)$ & 81.2 & $28.5(25.0-32.0)$ & $6,837,056$ & $1,946,038$ & $40.70(35.71-45.78)$ \\
\hline \multirow[t]{2}{*}{ Medication $^{\mathrm{a}}$} & 21,628 & $1.08(1.06-1.10)$ & 36.7 & $2.9(2.2-4.9)$ & $28,972,894$ & 826,382 & $38.20(28.85-65.45)$ \\
\hline & & & & Total & $107,282,173$ & $27,213,543$ & $\begin{array}{l}569.08(467.93- \\
672.79)\end{array}$ \\
\hline
\end{tabular}

$C I$ confidence interval, $C A N \$$ Canadian dollars, $P I P$ potentially inappropriate prescription, IRR incidence rate ratio, ED emergency department, STOPP Screening Tool of Older People's Prescriptions, START Screening Tool to Alert Doctors to Right Treatment

${ }^{a}$ Includes only those with a STOPP criterion as a their first PIP. All participants with a START criterion as their first PIP, multiple first PIP, or an overlapping second PIP within their 90-day outcome observation window were removed and the prevalence was recalculated

${ }^{\mathrm{b}}$ Costs attributable to PIP were determined by multiplying unrounded population attributable fractions by the total costs

visits. While this research was conducted using a cohort of residents of Ontario, due to the large cohort size spanning a diverse region of Canada, these results are likely to be generalizable to other provinces in Canada, as well as countries with similar healthcare systems and populations. The proportion of expenditures due to PIP identified in this study provide further evidence for PIP as a high priority target for intervention at the population-level in Canada and in other jurisdictions where PIP is prevalent. This study provides evidence on the total burden of PIP that could help decision makers with priority setting when PIP is compared with other important healthcare issues. Additionally, this study highlights the need for macro, health-system-level interventions to address PIP given its large burden and the fact that this burden is from a broad range of criteria in different disease areas and drug classes that might otherwise need to be addressed with micro-level, individual interventions. The PAF and costs related to PIP would decrease should the prevalence of PIP decrease, based on these interventions.

The subgroup and sensitivity analyses provide further characterization of the burden of PIP, and may provide some direction on the priorities for intervention. When assessing the health system costs due to PIP, by age, while PIP prevalence increased by age, the cost impact of PIP decreased with age due to decreasing IRRs, which indicate costs in the older populations are likely explained by other factors, such as comorbidity. Combined with their larger population size, this resulted in larger absolute costs in the younger age group, providing evidence for PIP interventions to be prioritized in younger seniors, as interventions targeting this population might have a greater impact. Additionally, the sensitivity analysis assessing the impact of the first PIP ever, with no other concurrent or overlapping PIPs, displays the importance of intervening as soon as possible on PIP. The primary analysis results show the average impact of all PIPs on outcomes, while the first PIP-only results show how vital it may be to intervene on the first PIP.

To the best of our knowledge, this is the first study of its kind to assess the burden of PIP beyond just medication costs at the population-level using linked health administrative data. Our findings of increased medication expenditures due to PIP are in line with previous research, although these studies only assessed the costs of a medication responsible for PIP, and did not include hospitalization or ED visits and also excluded costs related to newly prescribed medications that may have resulted from PIP, i.e. a prescribing cascade [8-10]. One of these studies assessed the cost of PIP in Canada using a subset of the Beers list, which previous research has shown to be not as sensitive at identifying PIP than the STOPP/START criteria [6], and found \$419 million was spent on potentially inappropriate medications outside of hospital settings in 2013 [10]. The other two studies were conducted in European settings with different observation periods, smaller subsets of the STOPP/START criteria, and smaller populations than our study. The application of 30 STOPP criteria to a national prescribing database in Ireland in 2007 identified total PIP medication expenditure to be $€ 39$ million, while the application of 28 STOPP criteria to a national primary care prescribing database in Northern Ireland in 2009/2010 observed $€ 6$ million in total medication expenditure as a result of PIP [8, 9].

Our study is subject to certain limitations around the methodology and data used. We used the PAF, which has several key assumptions, i.e. that the effect of PIP is reversible, interventions exist to address it, and the relationship between PIP and the outcomes assessed is causal [41]. The assumption with the greatest impact on the interpretation and validity of our results is that of causality, as observational studies such as ours typically only identify associations between an exposure and outcome of interest at best. 
While the relationship between PIP and the outcomes of interest addresses a number of the traditional Hill's criteria for assessing causal relationships [42], we cannot say for certain that PIP causes hospitalization, ED visits and additional prescribing of medications.

One other limitation related to this is the issue of residual confounding. Since our data source was not initially intended for research, we were limited by the availability of variables for the control of confounding within our models. While we are confident that the covariates used addressed much of the confounding by indication, it is possible that there is still some leftover confounding by unmeasured variables. Future work that would serve to potentially address these two limitations would be a study where hospitalizations are separated by whether they were planned or unplanned. This present study included all hospitalizations, but there are likely to be some planned hospitalizations not causally linked to PIP that were captured within the study that would contribute to confounding our results. It should also be noted that the use of the STOPP/START criteria in health administrative databases, compared with clinical data, in Ontario is currently being validated. While there is the potential for low sensitivity and specificity to detect PIP in HAD from these codes, the process used to identify PIP criteria applicable to HAD was conservative and transparent [37]. Additionally, since we are using health administrative data, not clinical data, and a conservative approach to identifying PIP was used, some PIPs cannot be identified, therefore we are thus likely to be underestimating the prevalence of PIP [6]. Given prevalence is an important factor in the PAF, this indicates that we may be underestimating the impact of PIP.

One important strength of this study to note is the method used to assign a random index date to participants without PIP. An unbiased approach to identifying a 90-day observation period for outcome ascertainment was needed, and the use of the distribution of time-to-PIP from accrual into the study cohort from patients with PIP, and applying it to patients without PIP, afforded us with the best solution. This may be useful in future drug safety and effectiveness studies facing a similar situation.

\section{Conclusions}

PIP in older adults is not only common and costly with regard to medication expenditures but it is also a significant source of costs from downstream events such as hospitalizations and ED visits. Future work should focus on identifying strategies and priorities for intervention at both the health system and individual levels. Younger seniors are at the highest risk of hospitalization and ED visit from PIP and have the highest cost burden, indicating that they may be a subgroup of interest when identifying populations where intervention may be most effective. Additional work should be conducted to identify priority PIPs that are most highly associated with negative burden and costs, given a lack of current health system-level interventions to address all PIP. Intervening on PIP would not only lead to savings on prescription drug costs but would also provide a health benefit to patients while significantly reducing downstream health system costs.

Author Contributions $\mathrm{CDB}$ is the principal author of this manuscript. The concept and methods for the study were conceived by CDB, LMB, KT and DC. Funding was secured by LMB (CIHR Grant Number 287245-HPM-BRUY-46830). Cohort creation, as well as exposure and outcome ascertainment, was conducted by CDB with the help of Glenys Smith from ICES. CDB analyzed data with assistance from KT. All listed authors provided feedback, help with revisions and approval of the final version of this manuscript.

Data Availability Statement The data set from this study is held securely in coded form at ICES. While data sharing agreements prohibit ICES from making the data set publicly available, access may be granted to those who meet prespecified criteria for confidential access, available at www.ices.on.ca/DAS. The full data set creation plan and underlying analytic code are available from the authors upon reasonable request, understanding that the programs may rely on coding templates or macros that are unique to ICES. This study was supported by ICES, which is funded by an annual grant from the Ontario Ministry of Health and Long-Term Care (MOHLTC). The opinions, results and conclusions reported in this paper are those of the authors and are independent from the funding sources. No endorsement by ICES or the Ontario MOHLTC is intended or should be inferred.

\section{Compliance with Ethical Standards}

Funding This work was supported by the Canadian Institutes of Health Research (CIHR), Grant Number 287245-HPM-BRUY-46830 (principal investigator: Lise M. Bjerre). Additionally, Cody Black is supported by a CIHR Drug Safety and Effectiveness Cross-Disciplinary Training programme traineeship and a University of Ottawa Excellence Scholarship.

Conflict of interest Cody Black, Kednapa Thavorn, Doug Coyle and Lise M. Bjerre report no conflicts of interest.

Ethical approval Ethical approval for this study was obtained from the Institutional Review Board at Sunnybrook Health Sciences Centre, Toronto, ON, Canada.

Open Access This article is distributed under the terms of the Creative Commons Attribution-NonCommercial 4.0 International License (http://creativecommons.org/licenses/by-nc/4.0/), which permits any noncommercial use, distribution, and reproduction in any medium, provided you give appropriate credit to the original author(s) and the source, provide a link to the Creative Commons license, and indicate if changes were made. 


\section{References}

1. Kaufmann C, Tremp R, Hersberger K, et al. Inappropriate prescribing: a systematic overview of published assessment tools. Eur J Clin Pharmacol. 2014;70:1-11.

2. Laroche ML, Charmes JP, Nouaille Y, et al. Is inappropriate medication use a major cause of adverse drug reactions in the elderly? Br J Clin Pharmacol. 2007;63(306):177-86.

3. Spinewine A, Schmader KE, Barber N, et al. Appropriate prescribing in elderly people: how well can it be measured and optimised? Lancet. 2007;370(1474):173-84.

4. O'Mahony D, O'Sullivan D, Byrne S, et al. STOPP/START criteria for potentially inappropriate prescribing in older people: version 2. Age Ageing. 2015;44(2):213-8.

5. American Geriatrics Society 2015 Beers Criteria Update Expert Panel. American Geriatrics Society 2015 Updated Beers criteria for potentially inappropriate medication use in older adults. J Am Geriatr Soc. 2015;63(11):2227-46.

6. Bjerre L, Halil R, Catley C, et al. Identifying potentially inappropriate prescribing in whole populations: coding the STOPP/ START and Beers criteria for use with large, routinely collected population datasets. 2018 (unpublished observations).

7. Bjerre L, Halil R, Catley C, et al. Assessing the association between potentially inappropriate prescriptions and patient outcomes at the population level: application of the STOPP-START and Beers criteria for use with large, routinely collected population datasets. 2018 (unpublished observations).

8. Cahir C, Fahey T, Teeling M, et al. Potentially inappropriate prescribing and cost outcomes for older people: a national population study. Br J Clin Pharmacol. 2010;69(5):543-52.

9. Bradley M, Fahey T, Cahir C, et al. Potentially inappropriate prescribing and cost outcomes for older people: a cross-sectional study using the Northern Ireland Enhanced Prescribing Database. Eur J Clin Pharmacol. 2012;68(10):1-9.

10. Morgan SG, Hunt J, Rioux J, et al. Frequency and cost of potentially inappropriate prescribing for older adults: a cross-sectional study. CMAJ Open. 2016;4(2):E346-51.

11. Stockl K, Le L, Zhang S, et al. Clinical and economic outcomes associated with potentially inappropriate prescribing in the elderly. Am J Manag Care. 2010;16(1):e1-10.

12. Black C, Thavorn K, Coyle D, et al. The health system costs of potentially inappropriate prescribing in Ontario, Canada: a protocol for a population-based cohort study. BMJ Open. 2018;8(6):e021727.

13. Benchimol EI, Smeeth L, Guttmann A, et al. The REporting of studies Conducted using Observational Routinely-collected health Data (RECORD) statement. PLoS Med (United States). 2015;12(10):e1001885.

14. Bronskill S, Carter M, Costa A, et al. Aging in Ontario: an ICES chartbook of health service use by older adults. Toroton: ICES; 2010.

15. Institute for Clinical Evaluative Sciences (ICES). Health Services Data. 2011. Available at: http://www.ices.on.ca/ webpage.cfm?site_id $=1 \&$ org_id $=26 \&$ morg_id $=0 \& g s e c$ $\mathrm{id}=5314 \&$ item $\_\mathrm{id}=5326$.

16. Institute for Clinical Evaluative Sciences. ICES Data Dictionary. 2016. Available from: https://datadictionary.ices.on.ca/Applicatio ns/DataDictionary/Default.aspx.

17. Institute for Clinical Evaluative Sciences. Datasets available through Data \& Analytic Services [cited 2 Feb 2018]. Available at: https://datadictionary.ices.on.ca/applications/datadictionary/ Default.aspx ?viewmode $=$ DataServices.

18. SAS Institute Inc. SAS ${ }^{\circledR} 9.4$ functions and CALL routines: reference. 5th ed. Cary, NC; SAS Institute, Inc.; 2016.

19. Wodchis W, Buchmeneva K, Nikitovic M, et al. Guidelines on person-level costing using administrative databases in Ontario.
Toronto, 2013. Available at: http://www.hsprn.ca/uploads/files/ Guidelines_on_PersonLevel_Costing_May_2013.pdf.

20. Statistics Canada. Consumer price indexes. 2017.

21. Boult C, Dowd B, McCaffrey D, et al. Screening elders for risk of hospital admission. J Am Geriatr Soc. 1993;41(8):811-7.

22. Anderson GF, Steinberg EP. Predicting hospital readmissions in the Medicare population. Inquiry. 1985;22(3):251-8.

23. Weissman J, Stern R, Epstein A. The impact of patient socioeconomic status and other social factors on readmission: a prospective study in four Massachusetts hospitals. Inquiry. 1994;31(2):163-72.

24. Krumholz HM, Parent EM, Tu N, et al. Readmission after hospitalization for congestive heart failure among Medicare beneficiaries. Arch Intern Med. 1997;157(1):99-104.

25. Chin M, Goldman L. Correlates of early hospital readmission or death in patients with congestive heart failure. Am J Cardiol. 1997;79(12):1640-4.

26. Hajjar ER, Cafiero AC, Hanlon JT. Polypharmacy in elderly patients. Am J Geriatr Pharmacother. 2007;5(4):345-51.

27. Bates DW, Miller EB, Cullen DJ, et al. Patient risk factors for adverse drug events in hospitalized patients. ADE Prevention Study Group. Arch Intern Med. 1999;159(0003-9926):2553-60.

28. Carey IM, De WS, Harris T, et al. What factors predict potentially inappropriate primary care prescribing in older people? Analysis of UK primary care patient record database. Drugs Aging. 2008;25(8):693-706.

29. Evans RS, Lloyd JF, Stoddard GJ, et al. Risk factors for adverse drug events: a 10-year analysis. Ann Pharmacother. 2005;39(7-8):1161-8.

30. Kanjanarat P, Winterstein AG, Johns TE, et al. Nature of preventable adverse drug events in hospitals: a literature review. Am J Health Syst Pharm. 2003;60(17):1750-9.

31. Dhalla IA, Anderson GM, Mamdani MM, et al. Inappropriate prescribing before and after nursing home admission. J Am Geriatr Soc. 2002;50(6):995-1000.

32. Phillips RS, Safran C, Cleary PD, et al. Predicting emergency readmissions for patients discharged from the medical service of a teaching hospital. J Gen Intern Med. 1987;2(6):400-5.

33. Reed RL, Pearlman RA, Buchner DM. Risk factors for early unplanned hospital readmission in the elderly. J Gen Intern Med. 1989;6(3):223-8.

34. Corrigan JM, Martin JB. Identification of factors associated with hospital readmission and development of a predictive model. Health Serv Res. 1992;27(1):81-101.

35. Colledge NR, Ford MJ. The early hospital readmission of elderly people. Scott Med J. 1994;39(2):51-2.

36. van Walraven C, Mamdani M, Fang J, et al. Continuity of care and patient outcomes after hospital discharge. J Gen Intern Med. 2004;19(6):624-31.

37. Bjerre L, Ramsay T, Cahir C, et al. Assessing potentially inappropriate prescribing (PIP) and predicting patient outcomes in Ontario's older population: a population-based cohort study applying subsets of the STOPP/START and Beers' criteria in large health administrative databases. BMJ Open. 2015;5(e010156).

38. Weaver C, Ravani P, Oliver M, et al. Analyzing hospitalization data: potential limitations of Poisson regression. Nephrol Dial Transpl. 2015;30:1244-9.

39. Levin M. The occurence of lung cancer in man. Acta Union Int Contra Cancrum. 1953;9:531-41.

40. Rockhill B, Newman B, Weinberg C. Use and misuse of population attributable fractions. Am J Public Health. 1998;88(1):15-9.

41. Levine B. What does the population attributable fraction mean? Prev Chronic Dis. 2007;4(1):A14.

42. Hill A. The environment and disease: association or causation. Proc R Soc Med. 1965;58:295-300. 